\title{
Performance of D-dimer for predicting sepsis mortality in the intensive care unit
}

\author{
Yan-Qiu Han', Li Yan², Lei Zhang1', Pei-Heng Ouyang1', Peng Li1', Giuseppe Lippi³, Zhi-De Hu*1 \\ ${ }^{1}$ Department of Laboratory Medicine, The Affiliated Hospital of Inner Mongolia Medical University, Hohhot, China \\ 2Department of Respiratory and Critical Care Medicine, The Affiliated Hospital of Inner Mongolia Medical University, Hohhot, China \\ ${ }^{3}$ Section of Clinical Biochemistry, University of Verona, Verona, Italy
}

*Corresponding author: hzdlj81@163.com

\begin{abstract}
Introduction: The prognostic value of D-dimer (DD) in sepsis remains controversial. This study aimed to investigate the performance of DD for predicting sepsis mortality in the hospital and for identifying its potential correlates.

Materials and methods: The clinical and laboratory data of adult sepsis patients were extracted from the Medical Information Mart for Intensive Care III (MIMIC III, v1.4) database using the structured query language (SQL). The database contains critical illness admitted to the intensive care unit at Beth Israel Deaconess Medical Center between June 2001 and October 2012. The association between DD and mortality was investigated with receiver operating characteristic (ROC) curve, restricted cubic spline and logistic regression analysis. Subgroup analysis was also used for identifying DD correlates.
\end{abstract}

Results: The study population consisted of 358 sepsis patients. Those who died during hospital stay ( $N=160$ ) had significantly higher DD values than those who survived $(N=198)$. The area under the ROC curve $(A U C)$ of DD was $0.59(P<0.010)$. In subgroup analysis, white blood cell (WBC) count $>18 \times 10^{9} / \mathrm{L}$ and vasopressor therapy significantly decreased DD diagnostic performance. Categorical DD value was independently associated with hospital mortality after sequential organ failure score (SOFA) and blood lactate adjustment. Restricted cubic spline analysis revealed a U-shape relationship between DD and in-hospital mortality.

Discussion: We conclude that the accuracy of DD for predicting in-hospital sepsis mortality depends on WBC count and vasopressor therapy. Both low and extremely elevated DD values are associated with higher risk of death.

Keywords: sepsis; fibrin/fibrinogen degradation products; intensive care units; treatment outcome; disseminated intravascular coagulation

\section{Introduction}

Sepsis is a major cause of morbidity and mortality in patients admitted to the intensive care unit (ICU). According to the Third International Consensus Definitions for Sepsis and Septic Shock (Sepsis-3) definition, sepsis is now considered as a "lifethreatening organ or system dysfunction, caused by a dysregulated host response to infection" (1). Among all types of biological dysregulations, haemostasis derangement is very frequent in septic patients (2). Disseminated intravascular coagulation (DIC) is a form of systemic blood clotting, mostly characterized by widespread activation of both platelets and coagulation cascade, leading to massive thrombin generation, eventually evolving towards diffuse thrombosis and multiple organ failure (MOF) (3). Recent evidence suggests that the prevalence of acute DIC in patients with sepsis may be comprised between $25-50 \%$, and its onset enormously magnifies the risk of death (4-7).

Unlike fibrin/fibrinogen degradation products (FDP), D-dimer (DD) is a specific degradation product of stabilized fibrin (8). Therefore, the presence of increased value of this biomarker reflects both thrombin generation, as well as fibrinolytic degra- 
dation. Several studies have addressed the potential prognostic value of DD in sepsis over the past decades, providing quite inconsistent or even controversial evidence. Although increased DD values have been associated with worse clinical outcomes in some studies, others failed to confirm such findings, revealing that the prognostic value of DD may be modest or poor in sepsis patients (914). Notably, a recent study has also demonstrated that sepsis patients with DD values within the normal reference range had a nearly 4-fold higher risk of dying than those with DD concentration modestly or markedly increased (15). Since confirmatory evidence would be needed to define the putative prognostic significance of measuring DD in patients with sepsis, as well as its potential clinical and laboratory correlates in this setting, we performed a retrospective study aimed to address the diagnostic performance of this biomarker in predicting in-hospital sepsis mortality.

\section{Materials and methods}

\section{Study design}

This retrospective study was based on an analysis of the Medical Information Mart for Intensive Care III (MIMIC III, v1.4) database. The structured query language (SQL) was used to extract data from MIMIC III, which is a freely accessible clinical database, encompassing a total number of 46,520 patients and 58,976 ICU admissions (16,17). All patients were admitted to the ICU at Beth Israel Deaconess Medical Center between June 2001 and October 2012. The database has been originally developed by the Massachusetts Institute of Technology (MIT), and all included patients have been de-identified to preserve their privacy. After having passed a mandatory examination on the website of the National Institutes Health (NIH), an author of this manuscript was allowed to obtain data from MIMIC III for research purposes. Informed consent is inherently waived due to data availability in form of the public database.

\section{Study population}

The inclusion criteria of this study were as follows: (i) age $>18$ years, (ii) definitive diagnosis of sepsis based on the International Classification of Diseases (ICD 9) code 995.92 (sepsis) and 785.52 (septic shock), which are currently recommended by the Sepsis-3, and (iii) at least one DD value measured within 24 hours after ICU admission (in patients with many sequential DD values available, the first one measured at ICU admission was selected). The plasma DD was assayed using HemosIL D-Dimer HS 500 kit on the Instrumentation Laboratory ACL TOP automated analyzer (Instrumentation Laboratory, Lexington, USA).

The following additional information was then extracted from the database: demographic characteristics, results of laboratory testing and blood gas analysis, severity scores, treatment modalities, comorbidity, length of stay in the ICU, and outcome (i.e., in-hospital death). All laboratory tests, except DD, were collected in the first 24 hours of patient admission. The sequential organ failure score (SOFA) and simplified acute physiology score (SAPSII) scores were calculated as previously described $(18,19)$.

\section{Statistical analysis}

The Kolmogorov-Smirnov test was used to verify the normal distribution of continuous data. Normal distributed continuous variables were expressed as mean and standard deviation (SD), and comparisons were made using Student's t test. Skewed data were instead expressed as median (and interquartile range; IQR) and comparisons were made using the Mann-Whitney $U$ test. Categorical data comparison was performed with the Chi-square test. The diagnostic accuracy of DD value for predicting in-hospital mortality was analysed with receiver operating characteristic (ROC) curve analysis, by calculation of the area under the curve (AUC) and its 95\% confidence interval (95\% $\mathrm{Cl}$ ), and with multivariate logistic regression models. A restricted cubic spline was used to analyse the relationship between DD and in-hospital mortality. All analyses were performed with $\mathrm{R}$ (version 3.5.0) and statistical significance was set at $P<0.05$. 


\section{Results}

\section{Characteristics of the study population}

The flowchart illustrating the population study selection is shown in Figure 1. A total of 358 patients could be included in this study, and their clinical and laboratory characteristics are summarized in Table 1. A 160/358 patients (44.7\%) died during hospitalization. Patients who died had significantly lower values of white blood cell (WBC) count, haemoglobin, platelets, and albumin. The values of bilirubin, blood lactate, activated partial thromboplastin time (APTT), prothrombin time (PT), international normalized ratio (INR), SOFA, and SAPSIl scores were also found to be significantly higher in patients who died. A larger prevalence of liver disease and coagulopathy could also be observed in patients who died, whilst the rate of patients who received renal replacement therapy (RRT), mechanical ventilation, and vasopressor therapy was also higher in patients who died.

\section{Performance of DD for predicting in-hospital mortality}

D-dimer values were found to be significantly higher in patients who died during hospital stay than in those who survived (Figure 2A). The ROC curve analysis, performed for evaluating the accuracy of DD values for predicting in-hospital mortality is shown in Figure 2B. The AUC of DD was 0.59 (95\% Cl, 0.53-0.65; $\mathrm{P}<0.010)$. The diagnostic accuracy of DD for predicting in-hospital mortality was also studied in some subgroups of patients, clustered according to their clinical characteristics, as shown in Figure 3. The AUC of DD in patients without vasopressor therapy was significantly higher than that in those undergoing treatment with these agents ( 0.75 vs. $0.55 ; \mathrm{P}=0.013$ ). The predictive accuracy of DD was also higher in patients with $\mathrm{WBC}<18 \times 10^{9} / \mathrm{L}$ than in those with $\mathrm{WBC}>18$ $\times 10^{9} / L$ (0.65 vs. 0.51; $\left.P=0.022\right)$.

Since a previous study reported that sepsis patients with normal DD concentration had worse outcome than those with increased values, we further compared in-hospital mortality between patients with normal (i.e., $<500 \mu \mathrm{g} / \mathrm{L}$ ), increased (i.e., between 500 and $4000 \mu \mathrm{g} / \mathrm{L}$ ) and extremely high (i.e., $>4000$ $\mu \mathrm{g} / \mathrm{L}) \mathrm{DD}$ values (15). The corresponding mortality rates in patients with normal, increased and extremely increased DD values were $0.60(9 / 15), 37.3 \%$ $(60 / 161)$ and $50.0 \%(91 / 182)$, exhibiting a statistical significance for trend ( $P=0.029$ by Chi-square test). Notably, the risk of death was also higher in patients with markedly increased DD values (i.e., > 4000 $\mu \mathrm{g} / \mathrm{L}$ ) than in those with modestly elevated concentrations (i.e., $500-4000 \mu \mathrm{g} / \mathrm{L}$, odds ratio (OD): 1.68 ; 95\% Cl: 1.09-2.59; $\mathrm{P}=0.020$ ).

In addition, we used the restricted cubic spline method to portray the relationship between DD and in-hospital mortality risk. As shown in Figure 4, a clear U-shape relationship could be observed between DD and the risk of in-hospital death. The

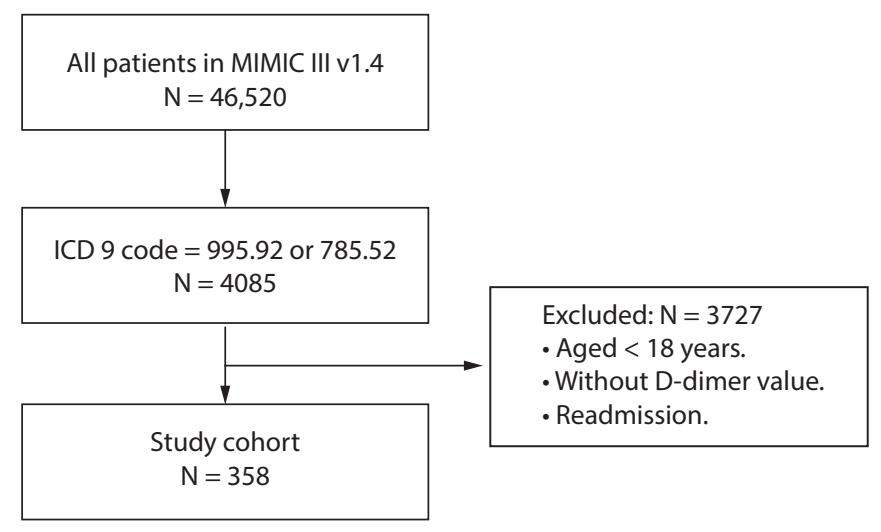

FIGURE 1. Flowchart of subjects' selection. MIMIC III - Medical Information Mart for Intensive Care III. ICD 9 - International Classification of Diseases. 
TABLE 1. Summary of the cohort

\begin{tabular}{|c|c|c|c|c|}
\hline Variables & $\begin{array}{c}\text { Total } \\
\mathbf{N}=\mathbf{3 5 8}\end{array}$ & $\begin{array}{c}\text { Alive } \\
N=198\end{array}$ & $\begin{array}{c}\text { Dead } \\
N=160\end{array}$ & $\mathbf{P}$ \\
\hline Age, years & $64(52-78)$ & $64(49-78)$ & $64(54-76)$ & NS \\
\hline Male, N (\%) & $196(55)$ & $100(51)$ & $96(60)$ & NS \\
\hline White, N (\%) & $244(68)$ & $133(67)$ & $111(69)$ & NS \\
\hline Emergency, N (\%) & $346(97)$ & $191(96)$ & $155(97)$ & NS \\
\hline WBC, $\times 10^{9} / \mathrm{L}$ & $16.0(8.6-24.0)$ & $17.7(11.2-26.1)$ & $13.5(5.8-21.1)$ & $<0.001$ \\
\hline Haemoglobin, g/L & 113 (101-127) & $116(105-131)$ & $110(98-123)$ & 0.002 \\
\hline Platelet, $\times 10^{9} / \mathrm{L}$ & $160(88-254)$ & $182(122-268)$ & $128(70-225)$ & $<0.001$ \\
\hline Albumin, $g / L$ & $28(23-31)$ & $28(24-32)$ & $27(23-31)$ & 0.049 \\
\hline Bilirubin, $\mu \mathrm{mol} / \mathrm{L}$ & $22(9-56)$ & $15(9-43)$ & $33(14-75)$ & $<0.001$ \\
\hline Creatinine, $\mu \mathrm{mol} / \mathrm{L}$ & 159 (106-248) & 159 (97-265) & $168(115-239)$ & NS \\
\hline Lactate, $\mathrm{mmol} / \mathrm{L}$ & $3.9(2.2-6.7)$ & $3.3(2.0-5.0)$ & $5.2(2.8-8.7)$ & $<0.001$ \\
\hline APTT, s & $\begin{array}{c}45.6 \\
(35.3-68.4)\end{array}$ & $\begin{array}{c}40.6 \\
(33.6-58.4)\end{array}$ & $\begin{array}{c}51.0 \\
(39.7-74.0)\end{array}$ & $<0.001$ \\
\hline INR & $1.9(1.5-3.1)$ & $1.7(1.4-2.3)$ & $2.3(1.7-3.7)$ & $<0.001$ \\
\hline PT, s & $\begin{array}{c}19.0 \\
(16.1-25.2)\end{array}$ & $\begin{array}{c}17.3 \\
(15.6-21.3)\end{array}$ & $\begin{array}{c}20.8 \\
(17.2-30.3)\end{array}$ & $<0.001$ \\
\hline$D D, \mu g / L$ & 4098 (1683-7828) & 3393 (1501-6835) & $4580(2039-8311)$ & 0.006 \\
\hline SOFA & $9(7-12)$ & $8(5-11)$ & $11(8-13)$ & $<0.001$ \\
\hline SAPSII & $51.44 \pm 16.85$ & $45.28 \pm 15.45$ & $59.07 \pm 15.35$ & $<0.001$ \\
\hline CHF, N (\%) & $135(38)$ & $72(36)$ & $63(39)$ & NS \\
\hline Cardiac arrhythmias, N (\%) & $139(39)$ & $79(40)$ & $60(38)$ & NS \\
\hline Hypertension, N (\%) & $153(43)$ & $84(42)$ & $69(43)$ & NS \\
\hline Chronic pulmonary, N (\%) & $63(18)$ & $35(18)$ & $28(18)$ & NS \\
\hline Renal failure, N (\%) & $71(20)$ & $37(19)$ & $34(21)$ & NS \\
\hline Liver disease, N (\%) & $110(31)$ & $40(20)$ & $70(44)$ & $<0.001$ \\
\hline Coagulopathy, N (\%) & $198(55)$ & $99(50)$ & $99(62)$ & 0.032 \\
\hline $\mathrm{SO} 2, \%$ & $95(91-98)$ & $94(91-97)$ & $95(93-98)$ & NS \\
\hline $\mathrm{SPO} 2, \%$ & $98(95-100)$ & $98(95-100)$ & $98(95-99)$ & NS \\
\hline $\mathrm{PCO} 2, \mathrm{kPa}$ & $4.8(4.0-6.0)$ & $4.8(4.1-5.9)$ & $4.9(3.6-6.1)$ & NS \\
\hline RRT, N (\%) & $40(11)$ & $12(6)$ & $28(18)$ & 0.001 \\
\hline Vasopressors, N (\%) & $293(82)$ & $149(75)$ & $144(90)$ & $<0.001$ \\
\hline Ventilation, N (\%) & $249(70)$ & $116(59)$ & $133(83)$ & $<0.001$ \\
\hline Days of ICU, days & $4(2-9)$ & $5(3-9)$ & $3(1-8)$ & $<0.001$ \\
\hline Days of hospital, days & $9(5-16)$ & $10(7-18)$ & $5(2-12)$ & $<0.001$ \\
\hline Severe sepsis, N (\%) & $328(92)$ & $184(93)$ & $144(90)$ & NS \\
\hline Septic shock, N (\%) & $259(72)$ & $144(73)$ & $115(72)$ & NS \\
\hline
\end{tabular}

Data with normal distribution were expressed as mean and standard deviation and compared by Student's t test. Skewed data were expressed as median (interquartile range) and compared by Mann-Whitney U test. Categorical data were expressed as absolute number (percentage) and compared by Chi-square test. WBC - white blood cell. APTT - activated partial thromboplastin time. PT - prothrombin time. INR - international normalized ratio. DD - D-dimer. SOFA - sequential organ failure score. SAPSII - simplified acute physiology score. CHF - congestive heart failure. SO2 - oxygen saturation. SPO2 - pulse oximetry. PCO2 - partial pressure of carbon dioxide. RRT - renal replacement therapy. ICU - intensive care unit. NS - non-significant. 
A

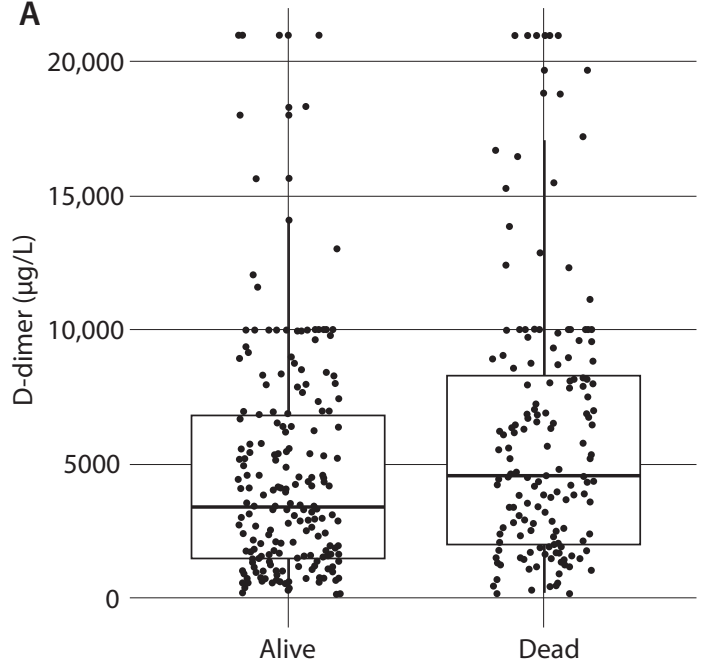

B

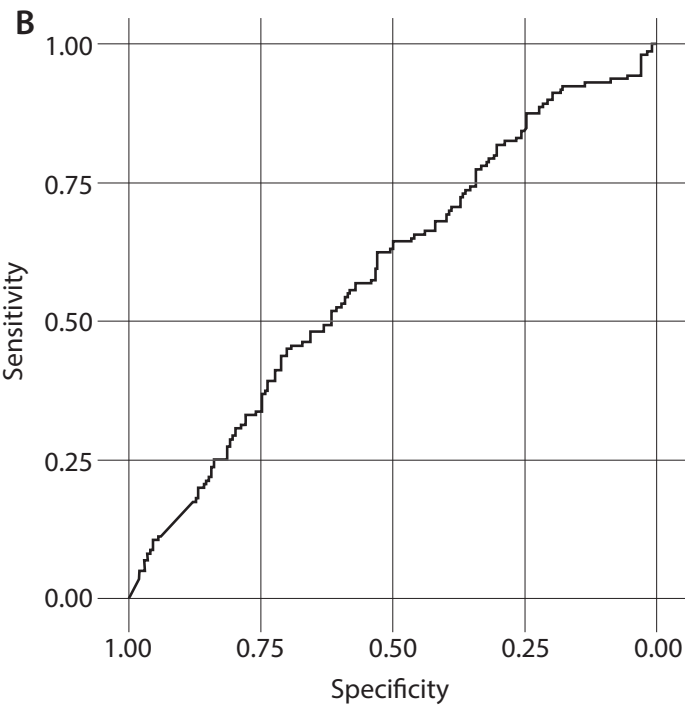

FIGURE 2. D-dimer predicts hospital mortality. A) Patients without hospital mortality had lower DD than those with hospital mortality. B) Receiver operating characteristic curve of DD for hospital mortality. DD - D-dimer.

DD concentration with the lowest risk of in-hospital mortality was found to be approximately 2700 $\mu \mathrm{g} / \mathrm{L}$.

\section{Multivariate analysis}

The results of logistic regression models carried out for evaluating the diagnostic accuracy of DD values in predicting in-hospital mortality are summarized in Table 2. Since some laboratory tests, especially platelet count and bilirubin, are included in the SOFA score, multiple adjustments were only carried out for SOFA and natural logarithm transformed lactate in our multivariate analysis. According to this analysis, natural logarithm transformed DD value was associated with in-hospital mortality in univariate statistics, whilst such association was lost in multivariate analysis. Next, we transformed DD as a categorical variable (i.e., $<500 \mu \mathrm{g} / \mathrm{L}, 500-$ $4000 \mu \mathrm{g} / \mathrm{L}$ and $>4000 \mu \mathrm{g} / \mathrm{L}$ ) (15). To facilitate results presentation, we set DD values $500-4000$ $\mu \mathrm{g} / \mathrm{L}$ as a reference. As shown in Table 2, the ODs of high DD $(>4000 \mu \mathrm{g} / \mathrm{L})$ and low DD $(<500 \mu \mathrm{g} / \mathrm{L})$ were larger than 1 , suggesting that both increased and decreased DD would be associated with a higher risk of in-hospital mortality.

\section{Discussion}

The identification of prognostic factors remains crucial in the management of sepsis, whereby treatment selection is currently considered the largest variable influencing patient outcomes. Some interesting findings have emerged from this study. First, we observed a U-shape relationship between DD values and in-hospital mortality in sepsis patients. The prognostic value of DD was found to be independent from the SOFA score, a widely used tool for predicting the outcome of sepsis. We then observed that the diagnostic accuracy of DD for predicting in-hospital sepsis mortality is largely dependent on WBC count and vasopressor therapy, whereby the prognostic value of this biomarker was higher in patients who did not receive vasopressors and with WBC count $<18 x$ $10^{9} / \mathrm{L}$. The results of this study hence seemingly suggest that more intensive treatment strategies would be necessary for patients with non-diagnostic (i.e., lower than the cut-off) or extremely elevated DD values. The most important conclusion emerging from this finding is that diagnosing DIC in sepsis patients using the criteria established by the International Society on Thrombosis and Hemostasis (ISTH) may be misleading, as suggested 


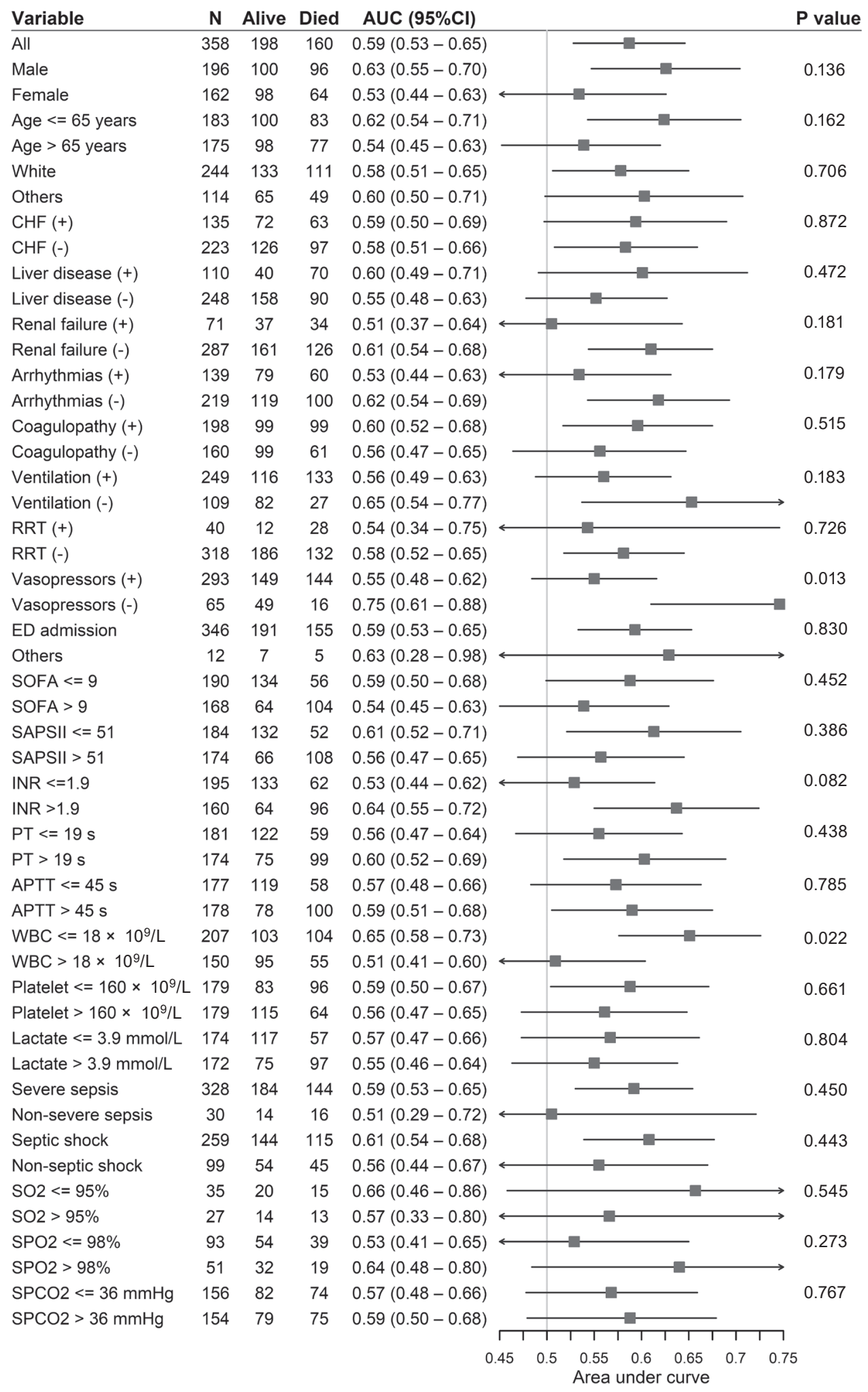

FIGURE 3. Forest plot display the area under curve of DD for hospital mortality. CHF - congestive heart failure. RRT - renal replacement therapy. ED - Emergency Department. SOFA - sequential organ failure score. SAPSII - simplified acute physiology score. INR - international normalized ratio. PT - prothrombin time. APTT - activated partial thromboplastin time. WBC - white blood cell. SO2 - oxygen saturation. SPO2 - pulse oximetry. PCO2 - partial pressure of carbon dioxide. 


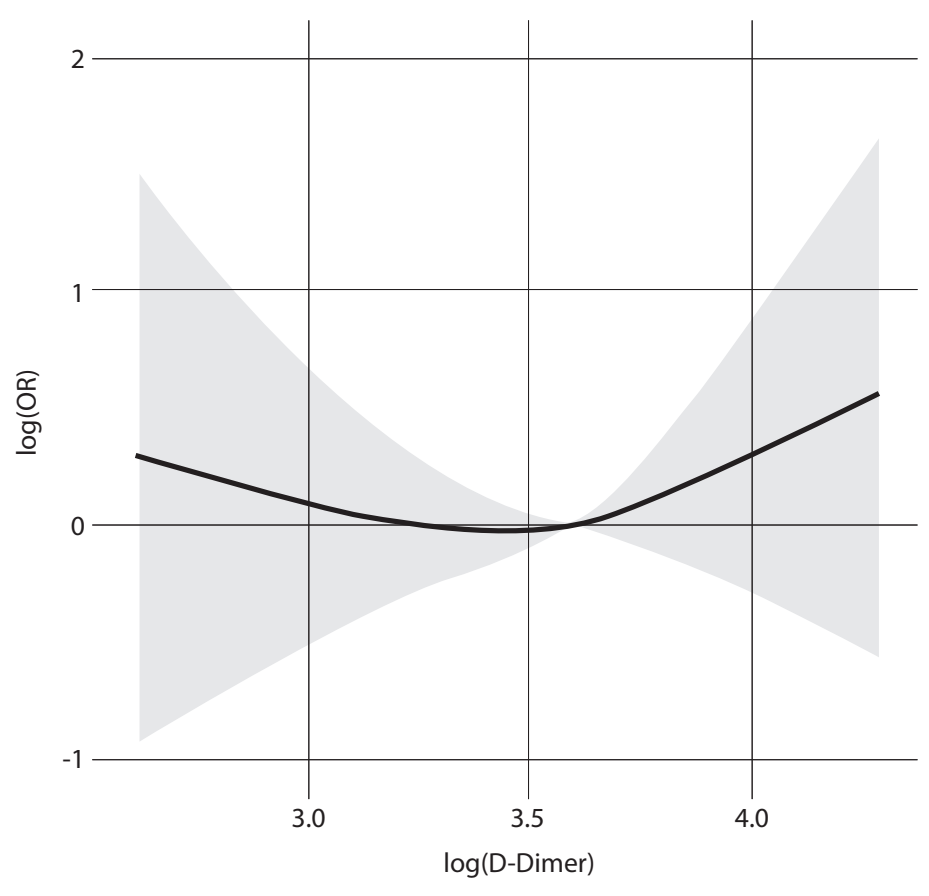

FIGURE 4. Restricted cubic spline reveals a U-shape relationship between DD and in-hospital mortality. OD - odds ratio. SOFA - sequential organ failure score.

TABLE 2. Prognostic value of DD with logistic regression models

\begin{tabular}{|c|c|c|c|c|}
\hline & \multicolumn{2}{|c|}{ Univariate } & \multicolumn{2}{|c|}{ Multivariate } \\
\hline & OR $(95 \% \mathrm{Cl})$ & $P$ & OR $(95 \% \mathrm{Cl})$ & $P$ \\
\hline $\operatorname{Ln}(D D)$ & $1.34(1.09-1.66)$ & 0.010 & $1.00(0.79-1.29)$ & NS \\
\hline$<500 \mu \mathrm{g} / \mathrm{L}$ & $2.53(0.87-7.86)$ & NS & $4.87(1.46-17.23)$ & 0.010 \\
\hline $500-4000 \mu \mathrm{g} / \mathrm{L}$ & 1 (Reference) & NA & 1 (Reference) & NA \\
\hline$>4000 \mu \mathrm{g} / \mathrm{L}$ & $1.68(1.10-2.60)$ & 0.020 & $1.05(0.64-1.74)$ & NS \\
\hline
\end{tabular}

OR - odds ratio. CI - confidence interval. NS - non-significant. NA - not applicable. Factors adjusted in multivariable logistic regression model are SOFA score and natural logarithm transformed lactate. DD - D-dimer.

by Semeraro and colleagues since we also observed that patients with non-diagnostic DD concentration may have up to $80 \%$ higher risk of death compared to those with values exceeding the diagnostic threshold $(15,20)$. Considering the retrospective nature of this study, characterized by modest sample size, future studies with prospective design and large sample size would be needed to validate our findings as well as those earlier published by Semeraro et al. (15).
Some hypotheses can also be brought in support of our findings. For example, the risk of mortality may be substantially enhanced in patients with prevalently thrombotic DIC subtypes, where fibrinolysis inhibition is exceptionally high, thus limiting thrombus (and fibrin) degradation, increasing disseminated thrombotic injuries, and ultimately preventing the increase of DD values in the bloodstream (15). 
This study has strength compared with previous investigations. First, the sample size $(\mathrm{N}=358)$ was higher than that of previous studies which have also attempted to define the prognostic value of DD in sepsis. Then, many earlier studies did not adequately address the potential clinical and laboratory correlates of $\mathrm{DD}$, which can ultimately influence its prognostic value. Notably, in the subgroup analysis carried out in our study, WBC count and vasopressor therapy were found to have an important impact in influencing the prognostic value of DD. The majority of previous studies also hypothesized the existence of a linear relationship between DD and sepsis mortality, whereby DD have been frequently associated with worse outcome. Nevertheless, in our additional analysis, where patients were stratified according to their DD values upon ICU admission as being normal $(<500 \mu \mathrm{g} / \mathrm{L})$, modestly increased (500-4000 $\mu \mathrm{g} / \mathrm{L})$ or markedly elevated ( $>4000 \mu \mathrm{g} / \mathrm{L}$ ), we found that patients with very low DD values paradoxically had the highest overall risk of death, up to $60 \%$.

These original findings can partially explain the heterogeneous evidence that emerged from previous studies which have also addressed the prognostic value of DD in sepsis. The vast majority of these previous investigations compared DD values in patients who died or survived, and then assessed its prognostic accuracy with ROC curve analysis (9-14). These two statistical methods would only be useful for biomarkers displaying a linear relationship with clinical outcome, since DD values distribution in cohort studies may have a remarkable influence on results. Moreover, the heterogeneous findings reported by previous studies may also be attributed to a lack of adjust- ment of final mortality analysis for WBC count and vasopressor treatment, which were instead important determinant of DD predictive accuracy in our study.

This study has some limitations, the first of which is the retrospective design. Then, DD value was unavailable for some sepsis patients within the first 24 hours upon ICU admission, and these subjects were hence excluded from our study. Finally, two ICD 9 codes (995.92 and 785.52) were used for identifying sepsis patients, which are highly specific, but less sensitive, for sepsis (21).

We could hence conclude that DD value may be an independent prognostic factor for in-hospital mortality in sepsis patients, and its prognostic accuracy is influenced by vasopressor therapy and WBC count. According to our data, a U-shape relationship can be postulated between DD values and in-hospital mortality. Both normal (i.e., $<500$ $\mu \mathrm{g} / \mathrm{L}$ ) and extremely elevated (i.e., $>4000 \mu \mathrm{g} / \mathrm{L}$ ) concentrations were associated with higher inhospital mortality. Considering that this is a retrospective single center study, further prospective, multicenter studies with a large sample size and a broader spectrum of sepsis patients would be needed to validate our findings.

\section{Acknowledgements}

The authors thank Alistair E.W. Johnson in Massachusetts Institute of Technology for providing details for the D-dimer assay.

\section{Potential conflict of interest}

None declared. 


\section{References}

1. Singer $M$, Deutschman CS, Seymour CW, Shankar-Hari $M$, Annane D, Bauer $M$, et al. The Third International Consensus Definitions for Sepsis and Septic Shock (Sepsis-3). JAMA. 2016;315:801-10. https://doi.org/10.1001/jama.2016.0287

2. Semeraro $N$, Ammollo CT, Semeraro F, Colucci M. Sepsis, thrombosis and organ dysfunction. Thromb Res. 2012; 129:290-5. https://doi.org/10.1016/j.thromres.2011.10.013

3. Levi $M$, Ten Cate $H$. Disseminated intravascular coagulation. N Engl J Med. 1999;341:586-92. https://doi.org/10.1056/NEJM199908193410807

4. Voves C, Wuillemin WA, Zeerleder S. International Society on Thrombosis and Haemostasis score for overt disseminated intravascular coagulation predicts organ dysfunction and fatality in sepsis patients. Blood Coagul Fibrinolysis. 2006;17:445-51. https://doi.org/10.1097/01. mbc.0000240916.63521.2e

5. Zeerleder S, Hack CE, Wuillemin WA. Disseminated intravascular coagulation in sepsis. Chest. 2005;128:2864-75. https://doi.org/10.1378/chest.128.4.2864

6. Patel $P$, Walborn $A$, Rondina $M$, Fareed J, Hoppensteadt D. Markers of Inflammation and Infection in Sepsis and Disseminated Intravascular Coagulation. Clin Appl Thromb Hemost. 2019;25:1076029619843338. https://doi. org/10.1177/1076029619843338

7. Gando S, Shiraishi A, Yamakawa K, Ogura H, Saitoh D, Fujishima $S$, et al. Role of disseminated intravascular coagulation in severe sepsis. Thromb Res. 2019;178:182-8. https://doi. org/10.1016/j.thromres.2019.04.025

8. Tripodi A. D-Dimer Testing in Laboratory Practice. Clin Chem. 2011;57:1256-62. https://doi.org/10.1373/ clinchem.2011.166249

9. Yin $Q$, Liu B, Chen $Y$, Zhao Y, Li C. The role of soluble thrombomodulin in the risk stratification and prognosis evaluation of septic patients in the emergency department. Thromb Res. 2013;132:471-6. https://doi.org/10.1016/j.thromres.2013.08.011

10. Li D, Zhou Y, Yu J, Yu H, Xia Y, Zhang L, et al. Evaluation of a novel prognostic score based on thrombosis and inflammation in patients with sepsis: a retrospective cohort study. Clin Chem Lab Med. 2018;56:1182-92. https://doi.org/10.1515/ cclm-2017-0863

11. Bouvier S, Cochery-Nouvellon E, Faillie JL, Lissalde-Lavigne G, Lefrant JY, Gris JC. Fibrin-related markers in patients with septic shock: individual comparison of D-dimers and fibrin monomers impacts on prognosis. Thromb Haemost. 2011;106:1228-30. https://doi.org/10.1160/TH11-07-0489
12. Innocenti F, Gori AM, Giusti B, Tozzi C, Donnini C, Meo F, et al. Prognostic value of sepsis-induced coagulation abnormalities: an early assessment in the emergency department. Intern Emerg Med. 2019;14:459-66. https://doi.org/10.1007/ s11739-018-1990-Z

13. Wang B, Chen G, Zhang J, Xue J, Cao Y, Wu Y. Increased Neutrophil Gelatinase-Associated Lipocalin is Associated with Mortality and Multiple Organ Dysfunction Syndrome in Severe Sepsis and Septic Shock. Shock. 2015;44:234-8. https://doi.org/10.1097/SHK.0000000000000408

14. Xu Z, Cheng B, Fu S, Liu X, Xie G, Li Z, et al. Coagulative biomarkers on admission to the ICU predict acute kidney injury and mortality in patients with septic shock caused by intraabdominal infection. Infect Drug Resist. 2019;12:2755-64. https://doi.org/10.2147/IDR.S218592

15. Semeraro F, Ammollo CT, Caironi P, Masson S, Latini R, Panigada $M$, et al. Low D-dimer levels in sepsis: Good or bad? Thromb Res. 2019;174:13-5. https://doi.org/10.1016/j.thromres.2018.12.003

16. Johnson AE, Pollard TJ, Shen L, Lehman LW, Feng M, Ghassemi $M$, et al. MIMIC-III, a freely accessible critical care database. Sci Data. 2016;3:160035. https://doi.org/10.1038/sdata.2016.35

17. Huang YL, Badrick T, Hu ZD. Using freely accessible databases for laboratory medicine research: experience with MIMIC database. J Lab Precis Med. 2017;2:31. https://doi. org/10.21037/jlpm.2017.06.06

18. Vincent JL, Moreno R, Takala J, Willatts S, De Mendonca A, Bruining $H$, et al. The SOFA (Sepsis-related Organ Failure Assessment) score to describe organ dysfunction/failure. On behalf of the Working Group on Sepsis-Related Problems of the European Society of Intensive Care Medicine. Intensive Care Med. 1996;22:707-10. https://doi.org/10.1007/ BF01709751

19. Le Gall JR, Lemeshow S, Saulnier F. A new Simplified Acute Physiology Score (SAPS II) based on a European/North American multicenter study. JAMA. 1993;270:2957-63. https:// doi.org/10.1001/jama.1993.03510240069035

20. Taylor FB, Jr., Toh CH, Hoots WK, Wada H, Levi $M$, et al. on behalf of the Scientific Subcommittee on Disseminated Intravascular Coagulation of the International Society. Towards definition, clinical and laboratory criteria, and a scoring system for disseminated intravascular coagulation. Thromb Haemost. 2001;86:1327-30. https://doi. org/10.1055/s-0037-1616068

21. Johnson AEW, Aboab J, Raffa JD, Pollard TJ, Deliberato $R O$, Celi $L A$, et al. A Comparative Analysis of Sepsis Identification Methods in an Electronic Database. Crit Care Med. 2018;46:494-9. https://doi.org/10.1097/ CCM.0000000000002965 